\title{
Vortices in Schwinger-Boson Mean-Field Theory of Two-Dimensional Quantum Antiferromagnets
}

\author{
Tai-Kai Ng \\ department of Physics, Hong Kong University of Science and Technology, \\ Clear Water Bay Road, Kowloon, Hong Kong
}

(April 1, 2018)

\begin{abstract}
In this paper we study the properties of vortices in two dimensional quantum antiferromagnets with spin magmitude $S$ on a square lattice within the framework of Schwinger-boson mean-field theory. Based on a continuum description, we show that vortices are stable topological excitations in the disordered state of quantum antiferromagnets. Furthermore, we argue that vortices can be divided into two kinds: the first kind always carries zero angular momentum and are bosons, whereas the second kind carries angular momentum $S$ under favourable conditions and are fermions if $S$ is half-integer. A plausible consequence of our results relating to the RVB theories of High- $T_{c}$ superconductors is pointed out.
\end{abstract}

PACS Nos, 75.10.Jm, 75.30.Ds, 79.60.-i

Typeset using REVTEX 


\section{INTRODUCTION}

In the past few years there has been a lot of interests in the study of quantum antiferromagnets in two dimension on a square lattice, stimulated by the discovery of High- $T_{c}$ superconductors [1,2]. Among others, one approximated approach to the quantum antiferromagnet problems is the Schwinger-Boson Mean-field Theory (SBMFT). [2]. In this approach, the quantum spins are represented as Schwinger bosons and an approximated ground state is constructed by bose condensation of spin-singlet pairs. The mean-field theory can also be formulated in a large-N expansion as the saddle point solution to a generalized $S U(N)$ quantum spin model [2, 3]. In the limit $N \rightarrow \infty$, the mean-field theory becomes exact. SBMFT predicts that in one dimension, Heisenberg antiferromagnet is always disordered, with a non-zero spin gap in the excitation spectrum. The theory is found to give an adequate description for integer spin chains, while it fails to describe the massless state of half-integer spin chains. This is because the topological Berry phase term which plays crucial role in the latter case is not taken into account correctly in SBMFT. [4] However, in two dimension topological term does not exist and SBMFT is more reliable. In particular, the theory predicts that the disordered (spin gap) phase exists only when the spin magnitude $S$ is small enough, when $S<S_{c} \sim 0.19$. It turns out that SBMFT offers a fairly accurate description for the magnetic properties of the High- $T_{c}$ compounds in the low doping regime [5]. However, at large doping levels, the generalization of SBMFT which includes holes as fermions [6], does not seem to describe the High- $T_{c}$ compounds correctly. For example, photo-emission experiments [7] indicate that the compounds have "large" Fermi-surfaces which satisfy Luttinger theorem, whereas the generalization of SBMFT to include holes predicts a "small" Fermi surface with area proportional to concentration of holes. In the large doping regime, it turns out that the slave-boson mean-field theory [8] which treats the spins as fermions and holes as bosons produces a lot of properties of High- $T_{c}$ compounds correctly 9 and the SBMFT is only superier in describing the low-doping, antiferromagnetic state. Thus a natural question is: what is the relation between the two theories? Can one understand the 
two theories within a single framework? How does nature crossover from one description to the other as concentration of holes increases?

In SBMFT, the spin operator $\vec{S}_{i}$ on site $i$ is expressed in terms of Schwinger bosons $\vec{S}_{i}=\bar{Z}_{i} \vec{\sigma} Z_{i}$, where $\vec{\sigma}$ is the Pauli matrix, $Z_{i}\left(\bar{Z}_{i}\right)$ are two component spinors $\bar{Z}_{i}=\left(\bar{Z}_{i \uparrow}, \bar{Z}_{i \downarrow}\right)$, etc. Notice that in order to represent a spin with magnitude $S$, there should be $2 S$ bosons per site [2]. The Hamiltonian can then be represented in terms of Schwinger bosons, and a mean-field theory can be formulated by introducing order parameters $\Delta_{i, i+\eta}=<Z_{i \uparrow} Z_{i+\eta \downarrow}-$ $Z_{i \downarrow} Z_{i+\eta \uparrow}>(\eta= \pm \hat{x}, \pm \hat{y})[$ [2]. Alternatively, SBMFT can also be formulated in a large-N expansion as the saddle point solution to a generalized $S U(N)$ quantum spin model. To derive the large-N theory, we divide the square lattice into $A$ and $B$ sublattices and consider the following transformation for the Schwinger bosons on $B$-sublattice,

$$
\begin{gathered}
Z_{j \uparrow}^{B}\left(\bar{Z}_{j \uparrow}^{B}\right) \rightarrow-Z_{j \downarrow}^{B}\left(\bar{Z}_{j \downarrow}^{B}\right), \\
Z_{j \downarrow}^{B}\left(\bar{Z}_{j \downarrow}^{B}\right) \rightarrow Z_{j \uparrow}^{B}\left(\bar{Z}_{j \uparrow}^{B}\right),
\end{gathered}
$$

for all sites $j$ on the $B$ sublattice. The Schwinger bosons on the $A$ sublattice remains unchanged in the above transformation. The Lagrangian of the Heisenberg model can then be represented in the transformed boson coordinates as [3]

$$
\begin{array}{r}
L=\sum_{i \sigma}\left[\bar{Z}_{i \sigma}^{A}\left(\frac{d}{d \tau}+i \lambda_{i}\right) Z_{i \sigma}^{A}-i 2 S \lambda_{i}\right]+\sum_{j \sigma}\left[\bar{Z}_{j \sigma}^{B}\left(\frac{d}{d \tau}+i \lambda_{j}\right) Z_{j \sigma}^{B}-i 2 S \lambda_{j}\right] \\
+J \sum_{i, \eta= \pm \hat{x}, \pm \hat{y}}\left|\Delta_{i, i+\eta}\right|^{2}-J \sum_{i \sigma, \eta= \pm \hat{x}, \pm \hat{y}}\left[\Delta_{i, i+\eta}^{*} Z_{i \sigma}^{A} Z_{i+\eta \sigma}^{B}+H . C .\right]
\end{array}
$$

where $\lambda_{i}$ 's are Lagrange multiplier fields enforcing the constraint that there are $2 S$ bosons per site, $\Delta_{i, i+\eta}$ 's are Hubbard- Stratonovich fields introduced in decoupling of $H . \sigma$ is the spin index. A $S U(N)$ spin theory can be formulated with the above Lagrangian if the usual $S U(2)$ spins $\uparrow$ and $\downarrow$ are extented to the $S U(N)$ case where N-spin species are introduced. SBMFT can be considered as the saddle point solution of the path integral over $L$ which becomes exact in the limit $N \rightarrow \infty$ [3]. For infinite system with no defects, the saddle point solution has position independent $\Delta_{i, i+\eta}$ 's and $\lambda$ 's and the solution is formally very 
similar to the BCS solution for superconductors, except that in present case the pairing objects are Schwinger bosons but not electrons. Another important difference is that in the present case, the pairing bosons are always located on different sublattices, whereas no such distinction is found in the case of superconductors.

The similarity between SBMFT and BCS theory leads us to ask the natural question of whether vortex-like solutions can be found in SBMFT as in the case of superconductors. More precisely, one may ask the question of whether one can construct a stable solution in SBMFT where $\Delta_{i, i+\eta}$ has a phase structure

$$
\Delta_{i, i+\eta} \sim\left|\Delta_{i, i+\eta}\right| e^{i \theta(i+\eta / 2)}
$$

where $\theta(\vec{x})$ is a smooth function of $\vec{x}$ and has a singular point at $\overrightarrow{x^{\prime}} \sim 0$ such that for distance $|\vec{x}|>>0, \theta(\vec{x}) \rightarrow \theta(\vec{x})+2 \pi$ if one moves vector $\vec{x}$ around a close loop $C$ which enclosed the point $\overrightarrow{x^{\prime}}=0$. As in the case of superconductors, the simplest way to address this question is to construct a continuum description for SBMFT (analogous to GinsburgLandau theory for superconductors) and study the possibility of having vortex solution in the continuum approximation. In the following we shall perform such a study in the disordered state in SBMFT where the Schwinger bosons are not bose-condensed and spin is a good quantum number. In realistic High- $T_{c}$ cuprates long range antiferromagnetic order is destroyed by introduction of charge carriers (holes). We shall not consider complications introduced by holes here and shall assume simply a disordered magnetic state with realistic spin magnitudes which can be described by the spin gap phase of SBMFT. We shall examine the properties of vortices within the continuum description. In section II we shall derive the continuum theory. Based on the continuum equations, we shall first study the properties of a single, non-magnetic impurity in the disordered state of SBMFT, where we shall point out the important difference between perturbations which are symmetric to the $\mathrm{A}$ and $\mathrm{B}$ sublattices, and perturbations which distinguish the two sublattices. In section III, we shall study static vortex excitations in our model. Based on the continuum theory, we shall argue that vortices are stable topological excitations in the disordered state of $2 \mathrm{D}$ quantum 
antiferromagnets. We shall then show that there exists two kind of vortices in the continuum theory, corresponding to vortices centered on the mid-point of a plaquette, and vortices centered on a lattice point. We shall show that the properties of these two kinds of vortices are very different, because of the different symmetry with respect to the two sublattices. The first kind of vortex which is symmetric to the two sublattices can carry only zero angular momentum and is a boson, whereas the second kind of vortex distinguishes between the two sublattices can carry angular momentum $S$ under favourble conditions and is a fermion if $S$ is half-integer. In section IV we shall concentrate ourselves at the case $S=1 / 2$ which is the physical case of interests and shall examine properties of a "liquid" of fermionic vortices. Based on simple symmetry arguments, we shall argue that the effective theory of a liquid of fermionic vortices has precisely the same form as the slave-boson mean-field theory for spins in the undoped limit. The case with finite concentration of holes will also be discussed. The findings in this paper will be summarized in section $\mathrm{V}$, where we shall discuss a plausible scenerio of how the system crossover from a state described by SBMFT to a state described by slave-boson mean field theory upon doping.

\section{CONTINUUM DESCRIPTION FOR SBMFT}

To derive the continuum theory we following Read and Sachdev [3] and consider the representation where our system has two sites per unit cell and introduce the 'uniform' and 'staggered' components in the $\Delta$ and $\lambda$ fields, where

$$
\Delta_{i, i \pm \eta}=\frac{1}{2}\left[\phi\left(i \pm \frac{\eta}{2}\right)+q_{ \pm \eta}\left(i \pm \frac{\eta}{2}\right)\right] e^{i\left[\theta\left(i \pm \frac{\eta}{2}\right)+A_{ \pm \eta}\left(i \pm \frac{\eta}{2}\right)\right]}
$$

where $q_{-\eta}=-q_{\eta}, A_{-\eta}=-A_{\eta}$ and in momentum space (for $\vec{k} \neq 0$ )

$$
\begin{aligned}
& \theta_{\tau}(\vec{k})=\frac{1}{2}\left[\lambda^{A}(\vec{k})+\lambda^{B}(\vec{k})\right], \\
& A_{\tau}(\vec{k})=\frac{1}{2}\left[\lambda^{A}(\vec{k})-\lambda^{B}(\vec{k})\right] .
\end{aligned}
$$


The $\phi(\vec{x})$ field describes uniform antiferromagnetic correlations whereas $q(\vec{x})$ field describes spin dimerization (spin-Peierls) effects [3]. $\theta(\vec{x})$ and $A_{\eta}(\vec{x})$ are fields describing the corresponding uniform and staggered phase fluctuations, respectively. All fields are slowly varying on the scale of a unit cell. Notice that the reason why four independent (real) fields are needed to describe fluctuations in one unit cell is precisely because we have divided the system into $A$ and $B$ sublattices. In the case of usual superconductors such a distinction is not present and only two real fields (or one complex scalar field) enters into the GinsburgLandau equation. Notice that $V_{\mu}(\vec{x})=\partial_{\mu} \theta(\vec{x})$ and $A_{\mu}(\vec{x})$ can be considered as $U(1)$ gauge fields coupling to the Schwinger boson $Z$ 's. The 'uniform' gauge field $V_{\mu}(\vec{x})$ couples to the bosons on the two sublattices with same gauge charge, whereas the gauge charges for the 'staggered' gauge field $A_{\mu}(\vec{x})$ are opposite on the two sublattices. A single vortex solution centered at $\overrightarrow{x^{\prime}}=0$ corresponds to a solution of SBMFT with boundary condition

$$
\oint V_{\mu}(\vec{x}) d x_{\mu}=2 n \pi
$$

or in terms of the gauge field, there is a 'uniform' gauge flux of $n / 2$ flux quantum passing through the origin, similar to the case of superconductors. Notice that in SBMFT where $\left\langle\Delta_{i, i+\eta}>\neq 0\right.$, the gauge symmetry associated with the 'uniform' gauge field $V_{\mu}(\vec{x})$ $\left(Z^{A} \rightarrow Z^{A} e^{i \Gamma}, Z^{B} \rightarrow Z^{B} e^{i \Gamma}, V_{\mu} \rightarrow V_{\mu}-2 \partial_{\mu} \Gamma\right)$ is broken, whereas the 'staggered' gauge symmetry $\left(Z^{A} \rightarrow Z^{A} e^{i \Gamma}, Z^{B} \rightarrow Z^{B} e^{-i \Gamma}, A_{\mu} \rightarrow A_{\mu}-\partial_{\mu} \Gamma\right)$ remains intact in SBMFT. The existence of vortex solution is tied with the broken symmetry gauge field $V_{\mu}(\vec{x})$ as in usual superconductors.

At distance much larger than lattice spacing, fluctuations associated with the 'uniform' variables $\phi$ and $\theta_{\tau}$ are unimportant. Thus we shall neglect the $\nabla \phi(x)$ and $\nabla \theta_{\tau}(x)$ terms in the following and derive a continuum theory for the rest of the variables. Notice that the $V_{\mu}$ variable is kept in our continuum theory since the term is essential for studying of vortices. In the continuum limit, the Lagrangian $L$ becomes

$$
L \rightarrow
$$




$$
\begin{aligned}
& \int d \tau \int d^{2} x\left\{\sum _ { \sigma } \left\{\bar{Z}_{\sigma}^{A}(x) \frac{\partial}{\partial \tau} Z_{\sigma}^{A}(x)+\bar{Z}_{\sigma}^{B}(x) \frac{\partial}{\partial \tau} Z_{\sigma}^{B}(x)\right.\right. \\
& -\sum_{\mu=\hat{x}, \hat{y}}\left[\phi(x)\left(1-\frac{1}{8}\left(V_{\mu}(x) V^{\mu}(x)\right)\right) \bar{Z}_{\sigma}^{A}(x) \bar{Z}_{\sigma}^{B}(x)-\frac{1}{2} \phi(x)\left(D_{\mu} \bar{Z}_{\sigma}^{A}(x)\right)\left(D_{\mu}^{*} \bar{Z}_{\sigma}^{B}(x)\right)\right. \\
& \left.+\frac{1}{2} q_{\mu}\left[\bar{Z}_{\sigma}^{B}(x) D_{\mu} \bar{Z}_{\sigma}^{A}(x)-\bar{Z}_{\sigma}^{A}(x) D_{\mu}^{*} \bar{Z}_{\sigma}^{B}(x)\right]+H . C .\right] \\
& +\theta_{\tau}(x)\left(\bar{Z}_{\sigma}^{A}(x) Z_{\sigma}^{A}(x)+\bar{Z}_{\sigma}^{B}(x) Z_{\sigma}^{B}(x)\right) \\
& \left.\left.+A_{\tau}(x)\left(\bar{Z}_{\sigma}^{A}(x) Z_{\sigma}^{A}(x)-\bar{Z}_{\sigma}^{B}(x) Z_{\sigma}^{B}(x)\right)\right\}+\frac{1}{2} \sum_{\mu}\left[\phi(x)^{2}+q_{\mu}(x)^{2}\right]-4 S \theta_{\tau}(x)\right\},
\end{aligned}
$$

where $D_{\mu}=\partial_{\mu}+i A_{\mu}$ and $D_{\mu}^{*}=\partial_{\mu}-i A_{\mu}$. The continuum Lagrangian Eq. (3) can be further simplified by introducing new boson fields

$$
\begin{aligned}
& \psi_{\sigma}=\frac{1}{2}\left(Z_{\sigma}^{A}+\bar{Z}_{\sigma}^{B}\right) \\
& \pi_{\sigma}=\frac{1}{2}\left(Z_{\sigma}^{A}-\bar{Z}_{\sigma}^{B}\right) .
\end{aligned}
$$

The $\pi_{\sigma}$ field can be integrated out safely at large distance and low energy [3], leaving effective Lagrangian for $\psi_{\sigma}$ field,

$$
\begin{aligned}
& L= \\
& \sum_{\sigma} \int d \tau \int d^{2} x\left\{\phi(x)\left(\left|D_{\mu}^{*} \psi_{\sigma}\right|^{2}+m(x)^{2}\left|\psi_{\sigma}\right|^{2}\right)+\left(\phi_{1}(x)\right)^{-1}\left(D_{\tau}-q_{\mu} D_{\mu}\right) \psi_{\sigma}^{+}\left(D_{\tau}^{*}+q_{\mu} D_{\mu}^{*}\right) \psi_{\sigma}\right. \\
& \left.+\frac{1}{2}\left[\phi(x)^{2}+q_{\mu}(x)^{2}\right]-4 S\left(2 \phi(x)\left(1-\frac{1}{8}\left(V_{\mu}(x) V^{\mu}(x)\right)\right)+m(x)^{2}\right)\right\}
\end{aligned}
$$

where $\phi(\vec{x}) m(\vec{x})^{2}=2 \theta_{\tau}(\vec{x})-4\left[1-(1 / 8)\left(V_{\mu}(\vec{x}) V^{\mu}(\vec{x})\right] \phi(\vec{x})\right.$ and and $\phi_{1}(\vec{x})=(1 / 2)\left\{\theta_{\tau}(\vec{x})+\right.$ $2\left[1-(1 / 8)\left(V_{\mu}(\vec{x}) V^{\mu}(\vec{x})\right] \phi(\vec{x})\right\}$. In the limit $q_{\mu}=0$ and $A_{\mu}=0$, and neglecting gradient terms $\nabla \phi, \nabla \theta_{\tau}$, the above Lagrangian can be easily diagonalized resulting in an effective Lagrangian $L_{\text {eff }}$ in terms of the $\phi(\vec{x})$ and $V_{\mu}(\vec{x})$ fields. The dynamical effect of the remaining terms can be obtained by looking at Gaussian fluctuations of the fields around the saddle point solution $A_{\mu}, q_{\mu}=0$. [3] In particular, in the small $m(\vec{x}) \sim m \rightarrow 0$ limit, we obtain (see Appendix)

$$
L_{e f f}=
$$




$$
\begin{aligned}
& \int d \tau \int d^{2} x\left\{(a-4(2 S+1)) \phi+\phi^{2}+\left(\frac{1}{2}-\frac{b}{\phi}\right) q_{\mu} q^{\mu}+\frac{(2 S+1) \phi}{2}\left(V_{\mu} V^{\mu}\right)\right. \\
& \left.+\frac{1}{2 e^{2}} F_{\mu \nu}^{2}+i c F_{\mu \tau} q_{\mu}+O\left(m^{2}\right)\right\}
\end{aligned}
$$

where $e^{2} \sim m, a(<4(2 S+1)), b$ and $c$ are constants of order $\mathrm{O}(1)$. The precise values of $a, b, c$ depend on the underlying lattice structure and cannot be obtained in a continuum theory. We have consider the realistic case $N=2$ in deriving the above expression. Notice that similar effective Lagrangian has been obtained by Read and Sachdev previously in studying effect of instantons [3]. The only difference here is that the $V_{\mu} V^{\mu}$ term which was not considered by Read and Sachdev is now retained. Recall that $V_{\mu}$ is the 'uniform' $U(1)$ gauge field coupling to the bosons and the $V_{\mu} V^{\mu}$ term in Eq. (5) just represents the Meissner effect associated with nonzero order parameter $\Delta$ in SBMFT. Notice that as in case of usual superconductors, we have choosen the London gauge $\nabla \cdot \vec{V}=0$ in our derivation. The term can also be written in a gauge invariant way by replacing $V_{\mu}$ by the gauge invariant object $V_{\mu}-2 \partial_{\mu} \Gamma$ in Eq. (5). This term does not contribute to the instanton effect discussed by Read and Sachdev [3] but is crucial to the study of vortices.

To understand more bout the continuum theory we first consider the properties of a single non-magnetic impurity in the disordered state of SBMFT. [10] We shall assume that a nonmagnetic impurity simply replaces a spin at site $i$ by a non-magnetic object. The simplest way to model this in our formalism is to is replace the constraint equation $\bar{Z} Z=2 S$ on site $i$ by $\bar{Z} Z=2 S\left(1-n_{i}\right)=0$, where $n_{i}$ is the number of non-magnetic impurity on site $i$. It is than easy to see that the effect of nonzero $n_{i}$ in our effective Lagrangian Eq. (5) is to introduce in the Coulomb gauge an extra term $-\left(2 S n_{i} e_{i}\right) A_{\tau}$, where $e_{i}= \pm 1$, depending on whether the impurity is located on the $A$ - or $B$-sublattices, [10] i.e., non-magnetic impurities appear as effective (staggered) gauge charges of magnitude $2 S e$ localized at the impurity sites. A corresponding electrostatic potential $V(r) \sim\left(2 S e_{i}\right) \ln (r / \xi)$ (where $\xi \sim m^{-1}$ ) will be induced around the impurity. To lower the electrostatic energy, $2 S$ bosons will be nucleated out from the vacuum to screen the electric field, resulting in formation of local magnetic moment of magnitude $S$ around a non-magnetic impurity [10. Notice that this effect has 
been observed experimentally in the High- $T_{c}$ compounds in the underdoped region upon substitution of $\mathrm{Cu}$ by $\mathrm{Zn}$ in the conducting planes [11].

This result can also be understood in an alternative way by observing that the same physical effect should have occurred if instead of replacing a spin on site $i$ by an non-magnetic impurity, we let the Heisenberg coupling $J_{i}$ to go to zero for those bonds joining to site $i$. In this case the 'impurity' site $i$ behaves as an non-magnetic impurity as far as the rest of the system is concerned. The only difference is that a free spin of magnitude $S$ now remains on site $i$. However, the effect of this approach on our effective Lagrangian Eq. (5) looks rather different compared with our previous approach. Instead of introducing a 'staggered' electric charge of magnitude $2 S e$ on site $i$, a boundary condition $\Delta_{i, i+\eta}=0(\eta= \pm \hat{x}, \pm \hat{y})$ is now imposed on our system. Using Eq. (2a), the corresponding boundary condition in the continuum theory becomes

$$
q_{\hat{n}}\left(x_{i}\right)= \pm \hat{n} \phi\left(x_{i}\right)
$$

where $i \rightarrow x_{i}$ in the continuum limit. $\hat{n}$ is a unit vector. \pm depends on whether site $i$ is on $A$ - or $B$ - sublattices. The properties of the system around the non-magnetic impurity is obtained by minimizing the free energy Eq. (5) under this boundary condition.

Performing the calculation, we find first of all that $\phi\left(x_{i}\right) \neq 0$ and correspondingly $\vec{q}\left(x_{i}\right) \neq$ 0. The reason for this behaviour can be understood easily from Eq. (5) by noticing that the coefficient in front of the $q_{\mu} q^{\mu}$ term becomes negative as $\phi$ becomes smaller than $2 b$, implying that spontaneously dimerization will occur when $\phi$ becomes small enough. Because of stability requirement, it can be shown that dimmerization $|\vec{q}|$ cannot have value larger than $q_{o}=(\sqrt{ } 2) \phi$ (see Appendix). Anyway both $\phi\left(x_{i}\right)$ and $\vec{q}\left(x_{i}\right) \sim \overrightarrow{q_{o}}$ should be nonzero in the continuum solution. The reason why $\vec{q}$ should be nonzero can also be understood from symmetry consideration. Notice that the $\phi(x)$ field is symmetric under exchange of $A$ and $B$ - sublattices whereas $q_{\mu}(x)$ field is antisymmetric. Thus a solution with $q_{\mu}(x)$ field identically zero would not distinguish between the two sublattices. However, the single nonmagnetic impurity problem certainly distinguishes the two sublattices since the impurity 
can only be placed on either one of the two sublattices. Thus we expect $q_{\mu}(x)$ to be nonzero around a single impurity. Minimizing the free energy with respect to the $q_{\mu}$ field we find also that $\vec{E}(x) \sim \vec{q}(x)$, where $E_{\mu}=i F_{\mu \tau}$ is the 'staggered' electric field in the system. With the boundary condition $\vec{q} \sim( \pm \hat{n}) \phi$ around the impurity we find that electric field radiating out (or in) from the impurity is obtained in our solution, i.e., depending on whether the impurity is located on the $A$ - or $B$ - sublattices, it behaves as source or sink for the electric field, in exact agreement with our previous approach which predicts that non-magnetic impurity behaves as a single staggered gauge charge added to the system.

One may also extend our approach to discuss the case when the Heisenberg coupling $J^{\prime}$ joining to the site $i$ is small but nonzero, so that the spin at site $i$ still couples weakly with the surrounding environment. The previous discussions still apply, except that the spin at site $i$ appears now as a localized spinon state occupied by $2 S$ spinons in the system (notice that at two dimension, an arbitrary small attractive interaction is enough to generate a bound state [12]). The spinon state again behaves as a (staggered) gauge charge (with magnitude $2 S$ ), and will bind $2 S$ other spinons with opposite gauge charge (or localized on opposite sublattice) next to itself as in the case with $J^{\prime}=0$. However for nonzero $J^{\prime}$ the localized spinons on the two sublattices will interact antiferromagnetically because of the underlying antiferromagnetic correlation in the system, [13] forming at the end a localized spin singlet around site $i$. It is only in the limit $J^{\prime} \rightarrow 0$ that the two spinons are decoupled and free local magnetic moments appear.

Our discussion can also be extended to study other forms of local defects. For example, one may study the situation when the Heisenberg coupling $J_{i, i+\hat{x}}$ between sites $i$ and $i+\hat{x}$ is set to zero, i.e., a single bond is being removed from the system. Notice that the perturbation is symmetric with respect to interchange of $A$ - and $B$ - sublattices. It is straightforward to generalize our previous discussion to this case where we find that the perturbation now appears as an effective electric dipole moment in $L_{\text {eff }}$. No local moment is expected to form around the impurity bond in this case because of the follow reason: because of the symmetry between $A$ - and $B$ - sublattices bosons(spinons) can be nucleated out from vaccuum only in 
pairs, with one localized on the $A$ sublattice and one on the $B$ sublattice. The interaction between the nucleated spinon pairs will again be antiferromagnetic and has a magnitude $\sim J e^{-x / \xi}$, where $x$ is the distance between the two spinons and $\xi \sim m^{-1}$ is the 'size' of the spinon wavefunction. For the single bond defect, the distance $x$ between the two spinons is of order of lattice spacing $<<\xi$. Thus the effective interaction between the two spinons is of order $J$, i.e., they will form a strong local spin singlet and no isolated magnetic moment will appear.

Experimentally, the $\mathrm{Cu}$ ion in the conducting plane of high- $T_{c}$ cupartes can be substituted by Zn (non-magnetic impurity) [1] and magnitude of Heisenberg exchange $J$ can be modified locally by introducing impurities out of conduction planes or substitution of $\mathrm{O}$ ions. It is observed that while substitution of $\mathrm{Cu}$ by $\mathrm{Zn}$ in the conducting plane introduces local magnetic moments in the underdoped (spin gap) phases of High- $T_{c}$ cuprates [11, no such effect is observed in other ways of introducing defects. Our theoretical investigation on disordered state of quantum antiferromagnets based on continuum description of SBMFT is in satisfying agreement with experimental results. [10]

\section{STATIC VORTICES IN DISORDERED STATE OF SBMFT}

In the continuum theory, a unit vortex centered as $\vec{x}=0$ corresponds to a solution of $\delta L_{\text {eff }}=0$, with $\theta(r, \Omega)=\Omega$, where $r$ and $\Omega$ are the distance and angle in the (2D) polar co-ordinate. The nonzero vorticity introduces a diverging kinetic energy through the $V_{\mu} V^{\mu} \sim(\nabla \theta)^{2}$ term in $L_{e f f}$, which supresses the magnitude of $\phi$ around the vortex core $r \rightarrow 0$, as in the case of usual superconductors. Minimizing the free energy with respect to $\phi$, and keeping in mind that for small $\phi,|\vec{q}| \sim(\sqrt{ } 2) \phi$, we find that $\phi=|\vec{q}|=0$ for $r<r_{o} \sim[(8(2 S+1)+4 b-2 a) /(2 S+1)]^{1 / 2}$, but not going to zero smoothly as $r \rightarrow 0$, as in the case of usual superconductors. This result is an artifact of our continuum theory where the $(\nabla \phi)^{2}$ term is not included in $L_{\text {eff }}$. Nevertheless, the qualitative effect where $\phi$ is being suppressed around the vortex core is clear. For large $r, \phi(r) \rightarrow \phi_{o}=(4(2 S+1)-$ 
a)/2 and $q \rightarrow 0$, indicating that vortices are stable topological excitations in SBMFT. The behaviour of vortices in SBMFT is very similar to vortices in usual superconductors at large $r$. However, the small $r$ behaviours are very different. The suppression of $\phi$ and $q$ fields around vortex core reflects the fact that around the center of vortex, the bond amplitude $\Delta_{i, i+\mu}$ 's are being suppressed. As has been discussed in the previous section, suppression of local bond amplitudes may lead to generation of localized magnetic moments, depending on the detailed bond configuration. To understand the properties of a vortex, it is thus important to understand the underlying bond structure at the vortex core.

Before studying the bond structure at vortex core, let us explain first how local magnetic moment binding to vortex core modifies the properties of a vortex. For a unit vortex the magnetic flux seen by a Schwinger boson is half flux quantum. (recall that $V_{\mu}$ is an 'uniform' gauge field which has same gauge charge for bosons on both sublattices) Therefore the orbital angular momentum of a Schwinger boson around a unit vortex is $\frac{1}{2}+$ integer. The composite object of one Schwinger boson bound to the vortex is thus a fermion because of the angularmomentum-statistics relation. [14] Notice that this phenomenon has a direct correspondence in superconductors where the bound state of an electron to a vortex is a boson. [14] However, if instead of one boson, two bosons are bound to a vortex, then the composite object is again a boson since angular momentum will be of integer value again. Now it should be clear why presence of local magnetic moment at vortex core is an importance issue. The magnitude of the magnetic moment measures the number of Schwinger bosons bound to the vortex and determines the statistics of the vortex.

The bond structure at the vortex core depends on where the center of vortex is being located. Here we shall consider only vortices which have maximum rotation symmetry allowed by the underlying lattice, i.e. rotation by $n \pi / 2$. In this case there are only two possible type of vortices, with vortex center located (i) at center of a plaquette and (ii) at a lattice point. (see Fig.1) We shall first consider vortex (i).

From continuum theory it is expected that the four bonds surrounding the center of vortex (see Fig.1a) will be largely suppressed because of the kinetic energy associated with 
the $V_{\mu} V^{\mu}$ term. Following similar analysis as in previous section we find that the suppressed bonds introduce in the continuum theory an effective electric quadrapole structure surrounding the vortex center. Notice that as in the case of single broken bond the structure is symmetric with respect to the $A$ - and $B$ - sublattices. Thus we expect that localized spinon pairs may form around the center of vortex but isolated magnetic moments cannot occur. Thus this kind of vortex carries zero angular momentum and is a boson.

The second kind of vortex is centered at a lattice point and as a result, it is expected that the four bonds joining to the vortex center (see Fig.1b) will be largely suppressed. This situation is very similar to the case of single non-magnetic impurity discussed in the last section. First of all, $2 S$ spinons will be found localized at the vortex center because of the suppressed bonds. The spinons behaves as a 'staggered' gauge charge with magnitide $2 S$ and $2 S$ other spinons with opposite gauge charge will be nucleated from the vacuum to screen the 'staggered' electric field generated by the spinons localized at the vortex center. The spinons on opposite sublattices interact antiferromagnetically through an effective Heisenberg exchange of order $J^{\prime} \sim J\left|\Delta_{c}\right|^{2}$, where $\Delta_{c}$ is the bond amplitude of the four bonds joining to the vortex center. As a result, a spin singlet will be formed. The vortex carries zero angular momentum and is again a boson. Notice that this is true even in the limit $J^{\prime} \rightarrow 0$ since integer number of bosons $=4 S$ are found binding to the vortex.

The situation is however quite different if we consider a finite density of the second kind of vortices. We shall show that when density of vortices is large enough, it may become energetically unfavourable to nucleate spinons from vacuum to screen the staggered gauge field and as a result, only $2 S$ spinons will be found binding to vortex center resulting in fermionic vortices when $S$ is half-integer (odd number of bound bosons). For simplicity we shall first consider two vortices seperated by distance $l$, with one vortex on each sublattice. First let us consider the energy when spinons are nucleated from the vacuum to screen the staggered gauge charge on each vortex. The total energy will be sum of three terms: (i) the electrostatic energy which is of order $2(2 S e)^{2} \ln \left(l_{o} / \xi\right)$, where $l_{o} \sim \xi$ is the 'size' of the nucleated spinon wavefunction, (ii) The exchange energy between the spinons localized as 
vortex center and nucleated spinons, which is of order $-2 S(S+1) J^{\prime} e^{-l_{o} / \xi} \sim-2 S(S+1) J^{\prime}$, and (iii) The energy needed to nucleate the spinons from the vacuum, which is of order $4 S m$. The sum of the three terms is of order $2 S\left(2 m-(S+1) J^{\prime}\right)$. The energy for the second case when no spinons are nucleated from vacuum consists of two terms: (i) the electrostatics energy which is of order $(2 S e)^{2} \ln (l / \xi)$ and (ii)the exchange energy between spinons located at the center of the two vortices which is or order $-S(S+1) J^{\prime} e^{-l / \xi}$. In $2 \mathrm{D}, e^{2} \sim m$ and the electrostatic energy is of order $4 S^{2} m \cdot \ln (l / \xi)$. For $l>>\xi$, it is certainly energtically more favourable to nucleate bosons from vacuum to screen the vortex gauge charge. However, when $l \leq \xi$, the energy cost of the second case is of order $-S(S+1) J^{\prime}$, and is energetically more favourable if $S(S+1) J^{\prime}<4 S m$. Notice that $J^{\prime} \sim J\left|\Delta_{c}\right|^{2}$ is expected to be very small because of suppresion effect around vortex core. Thus the condition can be satisfied easily even with a relatively small $m$. For finite density of vortices $\delta, l \sim \delta^{\frac{1}{2}}$. Thus for low concentration of vortices, we expect that spinons will be nucleated from vacuum to screen the staggered gauge field and the second kind of vortices are bosons. However, when density is high enough $\left(\delta \sim(1 / \xi)^{2}\right)$ and $m$ large enough, the situation will change and it may be energetically more favourable for the vortices to stay as fermions (for half-integer $S)$. Notice that finite density analysis for the first kind of vortices does not show similar qualitative difference between low and high density. Because of the symmetry between $A$ and $B$ - sublattice, bosons are always added or removed from a single vortex in pairs which cannot affect the statistics of a vortex.

Before ending this section let's summarize our findings here. Within a continuum description we have demonstrated in SBMFT the existence of vortex solutions which are stable topological excitations. We have found two different kind of vortices, according to the different locations of vortex center. The first kind of vortex has center located at center of a plaquette whereas the second kind of vortex has center located at a lattice point. The two kind of vortices have very different characters. The first kind of vortex is always a boson, whereas the second kind of vortex can be a fermion (for half-integer $S$ ) under conditions 
which are rather easy to acheive. We cannot, however, be absolutely sure whether 'fermionic' vortices exist because of the natural limitation of a continuum theory which gives only order of magnitude estimates. Notice also one important distinction between the vortices we study in this paper and usual vortices in superfluids. In the present case, vortices cannot be distingished by sign of vorticity since vortices carrying $1 / 2$ (or $\pi$ ) and $-1 / 2$ flux quanta should be considered as identical. On a lattice where the order parameter field $\Delta_{i, i+\mu}$ is a link-variable, $\pi$ and $-\pi$ vortices can be related by a pure gauge transformation. The usual singularity encountered in the gauge transformation in continous space does not arise here because of the discretized lattice structure.

It is important to clarify the different roles played by the 'uniform' and 'staggered' gauge fields in deciding the properties of vortices. The gauge fields arise from fluctuation in phases of the order parameter $\Delta_{i, i+\mu}$ 's. The uniform gauge field couples to bosons on the two sublattices with same gauge charge, and the corresponding gauge symmetry is broken in SBMFT. Vortices are stable because of this broken gauge symmetry, and corresponds to solutions with "uniform" magnetic flux penetrating center of vortices. The 'staggered' gauge field couples to bosons on opposite sublattices with opposite gauge charge. It plays no role in generating a stable vortex solution, but has strong effects in determining the precise properties of the vortex core. Without the 'staggered' gauge field, spinons need not be nucleated from vacuum to screen the 'staggered' charges generated at, for example, the center of the second kind of vortex. In this case, for half-integer spin systems, the second kind of vortices will always be fermions independent of density, since only odd number of bosons are found binding to center of each vortex (corresponding to the spin $S$ at center of vortex). Similarly, local magnetic moments will not be generated by non-magnetic impurities if 'staggered' gauge field does not exist.

It is also interesting to point out that similar vortex excitations have been considered by Read and Chakraborty [15] in a short-ranged RVB wavefunction for $S=1 / 2$ quantum antiferromagnets. They considered also the two kinds of vortices we discussed here. The statistics of the vortices were examined by direct Berry phase computations where similar 
conclusion that the first kind of vortex is a boson, whereas the second kind of vortex is fermion independent of density is arrived at their study. The effect of 'staggered' gauge field on vortices is not included in their study. The vortices we discuss in the present paper can be viewed as generalization of their vortices in short-ranged RVB wavefunction to the more complicated situation described by SBMFT.

\section{QUANTUM LIQUID OF FERMIONIC VORTICES}

In this section we shall examine the properties of a quantum liquid of fermionic vortices based on general symmetry considerations. We shall not ask the dynamical question of how the liquid of vortices come to exist in the first place but shall ask the simpler question if the liquid exists, what general properties should it has? For simplicity we shall restrict ourselves to the case of $S=1 / 2$ antiferromagnets which is the physical case of interests. In this case, a unit fermionic vortex carries spin $\pm 1 / 2$. Notice that vorticity does not contribute another independent quantum number as discussed in last section.

First of all we shall consider the Fock space for vortices. The 'vacuum' state for vortices is just a spin-disordered state in SBMFT. A one vortex state with spin $\sigma$ at position $i$ is a vortex centered at site $i$ with spin pointing in direction $\sigma$. Notice that two vortices with same $\sigma$ seperated by distance $d$ are not orthogonal to each other but have finite overlap $<\vec{x}_{1} \mid \vec{x}_{2}>\sim e^{-d / \xi}$. To construct orthogonal set of vortex states, we consider Wannier vortex states which can be constructed from vortex states using standard methods. The Wannier states are orthonormal to each other and represent localized vortices given that the overlap $<\vec{x}_{1} \mid \vec{x}_{2}>$ falls off exponentially as function of distance. [15]

Next we shall introduce second quantization representation for vortices. The creation operator $c_{i \sigma}^{+}$is defined as an operator which turns on an 'uniform' magnetic flux of $1 / 2$ flux quantum centered on site $i$, and creates a Schwinger boson with spin $\sigma$ on site $i$. Similarly, a destruction operator $c_{i \sigma}$ turns on an 'uniform' magnetic flux of $1 / 2$ flux quantum in opposite

direction, and removes a Schwinger boson with spin $\sigma$ on site $i$. With the constraint that we 
cannot put more than one Schwinger boson on any site, it can be checked easily that these operators obey usual fermion commutation rules. Notice that because of the constraint that there are always one Schwinger boson per site, a physical vortex cannot be created by $c_{i \sigma}^{+}$ operating on the 'vacuum' state, which changes the number of Schwinger bosons on site $i$. An example of an operator which creates a 'physical' vortex state from vacuum is $c_{i \sigma}^{+} Z_{i \sigma}$, where $Z_{i \sigma}$ is a Schwinger boson destruction operator with spin $\sigma$ on site $i$. Notice also that the vortex occupation number $\left\langle n_{i \sigma}^{v}\right\rangle=\left\langle c_{i \sigma}^{+} c_{i \sigma}>\right.$ is equal to the corresponding Schwinger boson occupation number $<n_{i \sigma}^{b}>=<\bar{Z}_{i \sigma} Z_{i \sigma}>$, since the only difference between vortex and boson operators is the magnetic flux which is being cancelled in the occupation number operator.

To proceed further we argue that the dynamics governing the quantum liquid of vortices must possess a local $S U(2)$ symmetry, where the Lagrangian for vortices must be invariant under the transformation

$$
\begin{array}{r}
c_{i \uparrow}^{+} \rightarrow \alpha_{i} c_{i \uparrow}^{+}+\beta_{i} c_{i \downarrow}, \\
c_{i \downarrow} \rightarrow-\beta_{i}^{*} c_{i \uparrow}^{+}+\alpha_{i}^{*} c_{i \downarrow},
\end{array}
$$

with $\left|\alpha_{i}\right|^{2}+\left|\beta_{i}\right|^{2}=1$. Notice that the same $S U(2)$ symmetry was obtained in the Heisenberg model for $S=1 / 2$ spins when the spins are expressed in terms of Fermion operators. 16, 17.

The reason why we expect the local $S U(2)$ symmetry to exist can be seen most easily by considering a physical vortex state with spin $\sigma$ on site $i$ and ask the question: what is the corresponding 'hole' or 'antiparticle' state for this vortex state? To construct the 'hole' state we operate $c_{i \sigma}$ on the 'vacuum' state, which creates a magnetic flux of $1 / 2$ flux quantum centered at site $i$, and remove a spin $\sigma$ Schwinger boson, leaving site $i$ empty. However, this is not a physically allowable state since the physically allowable state must has one boson per site. To construct a physical 'hole' state we must put back a Schwinger boson on site $i$. Now we may ask, which Schwinger boson shall we put back? It cannot be a spin $\sigma$ Schwinger boson, since putting back a spin $\sigma$ Schwinger boson just gives the original 'particle' vortex state. The only admissible choice is thus to put on site $i$ a spin $-\sigma$ boson, which creates a 
spin $-\sigma$ 'particle' vortex state. Thus we expect that the 'hole' state for a spin $\sigma$ vortex is a spin $-\sigma$ vortex, which gives rise to the local $S U(2)$ symmetry stated above. Another related argument is to observe that our underlying system is a pure spin system. Thus the effective vortex Hamiltonian must be equivalent to some effective pure spin Hamiltonian. The spin operator when expressed in terms of fermions possesses the $S U(2)$ symmetry stated above [16,17, so must be a pure spin Hamiltonian.

The $S U(2)$ local gauge invariance imposes a very severe constraint on the plausible form of the effective theory for the quantum liquid of vortices. A mean-field theory of a quantum liquid which is a spin singlet must describe a gas of fermions with particle-hole symmetry, i.e., a half-filled band with a particle-hole symmetric band-structure. Fluctuations around the mean-field theory must be described by a $S U(2)$ gauge theory. Physical excitations are represented by particle-hole excitations (generation of vortex pairs). These properties are all basic ingredients of the slave-boson-mean-field theory at half-filling, when holes are absent. 16,17 Notice in particular that the mean-field theory predicts that the Fermi surface for the vortices, if exists, must be 'large', i.e., it obeys the Luttinger theorem at half-filling, since $<n_{i \sigma}^{v}>=<n_{i \sigma}^{b}>=1 / 2$ for spin-singlet wavefunctions.

In the presence of holes the discussion is similar if one assumes that holes form bound state with vortices. Notice that in SBMFT where spins are bosons, holes are fermions and can be represented by 'slave-fermion' operators $f_{i}^{+}$and $f_{i}$. [6] A hole binding to a spin $\sigma$ vortex is formed by removing a spin $\sigma$ electron from the center of the spin $\sigma$ vortex. The resulting object is a boson, since it is formed by binding a fermion (hole) to a magnetic flux of 1/2 flux quantum. As in the case of vortices, we may introduce boson creation and destruction operators $b_{i}^{+}$and $b_{i}$ for the vortex-hole bound states. A boson creation operator $b_{i}^{+}$inserts a magnetic flux centered at site $i$, and create a (fermionic) hole on site $i$. Notice that as in the case of vortex operators, a physical hole cannot be created by operating $b_{i}^{+}$on the vacuum alone without first removing the spin on site $i$. An example of an operator which creates a physical vortex-hole bound state from vacuum is thus $b_{i}^{+} Z_{i \sigma}$, where the spin on site $i$ is removed by the $Z$ operator. Notice also that the vortex-hole bound state occupation 
number $<b_{i}^{+} b_{i}>$ is equal to the corresponding fermionic hole occupation number $<f_{i}^{+} f_{i}>$, since the only difference between the vortex-hole bound state operator and hole operator is the appearance of the magnetic flux, which is again cancelled in the occupation number operator.

In the presence of holes the $S U(2)$ symmetry associated with vortices will be broken. To see that we repeat our construction of the 'hole' state for a spin $\sigma$ vortex. In presence of holes the state generated by $c_{i \sigma}$ operating on the vacuum is a physical state, since now holes are allowed in the system. Thus the 'hole' state for a spin $\sigma$ vortex is not necessarily a spin $-\sigma$ vortex, and the $S U(2)$ symmetry is broken. The only symmetry which remains is the $U(1)$ symmetry $c_{i \sigma} \rightarrow c_{i \sigma} e^{i \theta}, b_{i}^{+} \rightarrow b_{i}^{+} e^{-i \theta}$, which follows from the requirement that a physical electron destruction operator is the product of the spin-annihilation operator and hole creation operator and that all physical processes must be expressed in terms of electron operators. Notice that because of the no double occupancy constraint $<n_{i \uparrow}^{v}>+<$ $n_{i \downarrow}^{v}>+<b_{i}^{+} b_{i}>=1$, a mean field theory which treats the vortices and vortex-hole bound states as independent gases of fermions and bosons, respectively will produce a vortex fermi surface (if exists) obeying the Luttinger theorem $<n_{i \uparrow}^{v}>=<n_{i \downarrow}^{v}>=(1-\delta) / 2$, where $\delta$ is the concentration of holes in the system, as in the slave-boson mean field theory for the $t-J$ model [8].

\section{CONCLUSION}

In this paper we study the properties of vortices in disordered state of SBMFT. Based on the continuum equations we show that vortices are stable topological excitations in the system. Furthermore, we show that because of the underlying lattice structure, there exists two kind of vortices with vortex center located at (i)center of a plaquette, and (ii)lattice point. The two kind of vortices have different properties because of the different symmetry with respect to the two sublattices. In particular, for half-integer spin systems, we argue that the second kind of vortices can be fermions, under conditions which are not difficult 
to achieve. We next consider a quantum liquid of fermionic vortices and show that because of general symmetry requirement, the effective theory for the liquid of vortices must has a general form very similar to the slave-boson mean field theory in the undoped limit. In the presence of holes similar conclusion is reached if one assumes that holes form bound states with vortices. A very interesting possibility brought out by our analysis is that the Schwinger-boson-slave-fermion mean field theory which is believed to describe the $t-J$ model qualitatively correctly in the $\delta \rightarrow 0$ limit and the slave-boson mean field theory which is believed to describe the metallic state more correctly can be understood within a single framework, if one identify the fermionic spins in the slave-boson mean field theory as vortices in Schwinger-boson treatment. In this paper we have demonstrate the possibility of such an identification. However, we have not been able to show within the framework of Schwingerboson-slave-fermion mean field theory that fermionic vortices and vortex-hole bound states are more important low energy excitations then bosonic spinwaves and fermionic holes when density of holes is high enough. The problem is related to the more fundamental question of how the antiferromagnetic spin background get disordered when holes are being introduced. We shall not try to address these questions further in this paper, except to point out lastly that based on the slave-fermion mean field theory for the $t-J$ model, fermionic vortices and vortex-hole bound states indeed become more stable in the presence of holes.

In the presence of holes, the effective Hamiltonian for the spins (in Schwinger-bosonslave-fermion treatment) has a form [6]

$$
\begin{aligned}
H= & -J \sum_{i, \eta}\left\{\Delta^{*}\left[Z_{i \uparrow}^{A} Z_{i+\eta \downarrow}^{B}-Z_{i \downarrow}^{A} Z_{i+\eta \uparrow}^{B}\right]+H . C .\right\} \\
& +\lambda \sum_{i \alpha}\left[\sum_{\sigma} \bar{Z}_{i \sigma}^{\alpha} Z_{i \sigma}^{\alpha}-(1-\delta)\right] \\
& +\sum_{i \sigma, \eta}\left\{\left(J Q^{*}+t F^{*}\right) \bar{Z}_{i \sigma}^{A} Z_{i+\eta \sigma}^{B}+H . C .\right\}
\end{aligned}
$$

where $\eta= \pm \hat{x}, \pm \hat{y}, \alpha=A, B, Q=\sum_{\sigma}<\bar{Z}_{i \sigma}^{A} Z_{i+\eta \sigma}^{B}>$ and $F=<f_{i}^{+} f_{i+\eta}>$. The first term is the original Heisenberg interaction and the $t F+J Q$ term is an effective hopping term for spins generated by motion of holes. The second term represents the constraint 
$\bar{Z}_{i} Z_{i}+f_{i}^{+} f_{i}=1$ satisfied on average. Notice that the effective Hamiltonian for Schwinger bosons still has a form very similar to the BCS Hamiltonian for superconductors. Thus we expect stable vortex solutions will exist. The most important difference in the presence of holes is the existence of the effective hopping term which breaks the staggered gauge symmetry. [18] The 'staggered' gauge charge is no longer a conserved quantity and as a result, the 'staggered' gauge field we discuss in the previous sections will no longer be important [18. In particular, the second kind of vortex will be more likely to stay as fermions since the 'staggered' electric field which is responsible for binding another boson to the vortex is no longer important. It is also easy to see that provided that fermionic vortices exist, it is energetically favourable to bind holes to them. The reason is simple: the antiferromagnetic correlation around the vortex core is suppressed by the $V_{\mu} V^{\mu}$ term and as a result, a hole can gain more kinetic energy by staying at the center of a vortex.

This work is supported by Hong Kong UGC Grant HKUST636/94P.

\section{APPENDIX A:}

In this appendix we outline our derivation leading to the effective Lagrangian Eq. (5). First we shall neglect the staggered gauge field $A_{\mu}$ and shall set the terms $\phi(x), q_{\mu}(x)$ and $V_{\mu}(x)$ equal to constants, i.e. we neglect gradient effects associated with these terms. In this case the effective Lagrangian Eq. (3) can be diagonalized easily leading to Free energy (at zero temperature)

$$
F=2 \sum_{\vec{k}} E(\vec{k})+\frac{1}{2}\left(2 \phi^{2}+\vec{q} \cdot \vec{q}\right)-2(2 S+1)\left(2 \phi\left(1-\frac{1}{8} \vec{V} \cdot \vec{V}\right)+m^{2}\right)
$$

where $E(\vec{k})=+\left(2 \phi^{2} \vec{k} \cdot \vec{k}-(\vec{q} \cdot \vec{k})^{2}+m^{2}\right)^{1 / 2}$ is the energy for the (bosonic) spinons, $m$ is the spin gap. The factor 2 is coming from spin sum. In the limit $m \rightarrow 0$ and $\vec{q} \rightarrow 0, E(\vec{k}) \rightarrow \sqrt{ } 2 \phi|\vec{k}|$ and $F \rightarrow(a-4(2 S+1)) \phi+\phi^{2}+(S+1 / 2) \phi\left(V_{\mu} V^{\mu}\right)$, where $a$ is a cutoff dependent constant coming from $\sum E(\vec{k})$. For $\vec{q}$ small, we can expand the $(\vec{q} \cdot \vec{k})^{2}$ term in $E(\vec{k})$ to obtain the $q_{\mu} q^{\mu}$ term in Eq. (5). Notice that $\phi$ is always a postive real number since the postive root 
is choosen in computing $E(\vec{k})$. Notice also that the stability criteria $E(\vec{k}) \geq 0$ for all values of $\vec{k}$ leads to the condition $\vec{q} \cdot \vec{q} \leq 2 \phi^{2}$. The terms involving the staggered gauge field $F_{\mu \nu}$ in Eq. (5) can be obtained by considering Gaussian fluctuations associated with the $A_{\mu}$ field. Detailed calculation can be found in reference [3] and we shall not repeat the derivation here. 


\section{REFERENCES}

[1] P.W. Anderson, Science 235, 1196(1987).

[2] A. Auerbach and D.P. Arovas, Phys. Rev. Lett. 61, 617(1988); D.P. Arovas and A. Auerbach, Phys. Rev. B 38, 316(1988); D. Yoshioka, J. Phys. Soc. Jpn. 58, 32(1989).

[3] N. Read and S. Sachdev, Phys. Rev. Lett. 62, 1694(1989); N. Read and S. Sachdev, Phys. Rev. B 42, 4568(1990).

[4] F.D.M. Haldane, Phys. Lett. 93A, 464(1983); I. Affleck, Nucl. Phys. B257, 397(1985).

[5] B. Keimer et. al., Phys. Rev. B 46, 14034(1992); S.W. Cheong et. al., Phys. Rev. Lett. 67, 1791(1991); see also R.J. Birgeneau and G. Shirane, in "Physical Properties of High Temperature Superconductors I", edited by D.M. Ginsberg, for a review.

[6] C.L. Kane, P.A. Lee, T.K. Ng, B. Chakraborty and N. Read, Phys. Rev. B 41, 2653(1990); B. Normand and P.A. Lee, preprint.

[7] C.G. Olson et. al., Phys. Rev. B 42, 381(1990); D.S. Dessau et. al., Phys. Rev. Lett. 71, 2781(1993); R. Liu et. al., Phys. Rev. B 46, 11056(1992).

[8] G. Baskaran, Z. Zou and P.W. Anderson, Solid State Commun. 63, 973(1987); C. Gros, R. Joynt and T. M. Rice, Phys. Rev. B 36, 8190(1987); G. Kotliar and J. Liu, Phys. Rev. B 38, 5142(1988).

[9] N. Nagaosa and P.A. Lee, Phys. Rev. Lett. 60, 2450(1990); P.A. Lee and N. Nagaosa, Phys. Rev. B 46, 5621(1992).

[10] N. Nagaosa and T.K. Ng, to appear in Phys. Rev. B .

[11] G. Xiao, M.Z. Cieplak, J.Q. Xiao, and C.L. Chien, Phys. Rev. B 35, 8782(1987); H. Alloul et. al., Phys. Rev. Lett. 67, 3140(1991); G. Zheng et. al., J. Phys. Soc. Jpn. 62, 2591(1993); K. Ishida et. al., J. Phys. Soc. Jpn. 62, 2803(1993); A.V. Mahajan, H. Alloul, G. Collins, and J.F. Marucco, Phys. Rev. Lett. 72, 3100(1994); P. Mendels et. 
al., Phys. Rev. B 49, 10035(1994).

[12] The existence of bound state for arbitrary weak attractive potential can be seen in continuum theory by directly solving the (continuum) mean-field eigenvalue equation $\left(-\nabla^{2}+m^{2}\right) \psi(\vec{x})-V_{o} \delta(\vec{x}) \psi(\vec{x})=E^{2} \psi(\vec{x})$ for arbitrary small $V_{o}$.

[13] M. Hagiwara, K. Katsumata, I. Affleck, B.I. Halperin, and J.P. Renard, Phys. Rev. Lett. 65, 3181(1990).; T. Kennedy, J. Phys. Condens. Matter 3, 5757(1990).

[14] F. Wilczek, Phys. Rev. Lett. 48, 1144(1982).

[15] N. Read and B. Chakraborty, Phys. Rev. B 40, 7133(1989).

[16] E. Dagotto, E. Fradkin and A. Moreo, Phys. Rev. B 38, 2926(1988).

[17] F.C. Zhang, C. Gros, T.M. Rice, H. Shiba and R. Joynt, Physica C153-155, 1251(1988).

[18] N. Nagaosa and P.A. Lee, Phys. Rev. B 45, 10719(1992). 


\section{FIGURES}

FIG. 1. two possible kind of vortices with vortex center located at (a) center of a plaquette, and (b)lattice point. In (a), the four bonds surrounding the vortex center and in (b), the four bonds joining to the vortex center, are suppressed. 